\title{
36th ANNUAL SASKATCHEWAN CHRISTMAS BIRD COUNT - 1977
}

Compiled by MARY I. HOUSTON, 863 University Drive, Saskatoon, Saskatchewan S7N $0 J 8$

As a result of the boundless enthusiasm of people such as Wayne Harris, Sheila Lamont, Wayne Renaud and Cuy Wapple, who separately and together organized counts in 15 localities over the Christmas season, we have an appreciable increase in the number of counts for 1977. Last year's high of 55 localities has been dwarfed by this year's 68. Also commendable was the very thorough report from Togo in which watchers at seven farms recorded their sightings around the yard, at the feeder or on trips in the neighborhood, for every day from December 21 to January 2 . These were compiled and submitted by Wanda May, one of the group, and have given us a representative picture of the winter birds of the area.

From the 66 species sighted this year (63 on count day and 3 additionals during count period) no new species were added to the all-time list, so the total remains at 123 for count day with 2 additionals for count period over the years.

Gray-crowned Rosy Finches were reported again this year from Fort Walsh, and also from EastendRavenscrag, Kindersley and White Bear. A Glaucous Gull was again reported, but from Cold River. Golden Eagles in 15 locations ( 8 in 1976 and 7 in 1975) and Bald Eagles in 8 locations ( 2 in 1976 and 3 in 1975) indicated an increase especially in the Cypress Hills area where 5 Colden and 4 Bald eagles were reported from Fort Walsh and 1 Colden and 2 Bald eagles from Skull Creek. Bohemian Waxwings were reported in large numbers from North Battleford and Saskatoon areas. White-breasted Nuthatches (14 locations) this year greatly outnumbered Red-breasted Nuthatches (2 locations). Evening and Pine Grosbeaks appeared much more frequently (28 in 1977, 12 in 1976) and (52 in 1977, 10 in 1976), respectively. Redpolls too, were common (53 localities) and in larger numbers than last winter.

This year the results have been organized into four tables: Table 1: participants; Table 2: methods of observation, weather and total individuals; Table 3: species and numbers from more than one locality on count day and in count period; Table 4: species recorded from only one locality on count day.

Two additional features have been included: temperature and snow data for seven localities for November and December as an indication of conditions leading up the Christmas counts (Table 5) and a summary of locations with the largest number of species in each of the last 26 years, compiled by Wayne Renand (Table 6).

Table 1. CHRISTMAS BIRD

\section{COUNT PARTICIPANTS}

BANGOR. Mrs. A. Thompson

BATTLEFORD-NORTH BATTLEFORD Pern Cordery, Heywood MacDonnell

BEAVER CREEK. Muriel Carlson, Rita Schilling

BESNARD LAKE. Arnold Nijssen, Tim Stacey, Douglas W. A. Whitfield

BICGAR. Don Renaud, Wayne Renaud, Adam Schmidt, Guy Wapple, Robert Wapple, Rodney Wapple

BIG GULLY CREEK. $(24 \mathrm{~km}$ NE of Maidstone). Wayne Harris and Sheila Lamont

BROADVIEW. Don Weidl, Dave Chaskavich

CANDLE LAKE. Wayne Harris, Sheila Lamont

CLARKBORO. Muriel Carlson, Mary Johnston 
COLD RIVER. Willie Haras

DALMENY. Ruth Purdy, Gilbert Sperling, Loyd Sperling (compiler), Mara Sperling

DILKE. Margaret Belcher (compiler), Mr. and Mrs. S. R. Belcher

DUCK LAKE. Bob Godwin, Kathy Meers.

DUPEROW-RUTHILDA. Paul de Bussac, Morris L'hoir, Guy Wapple, Rodney Wapple

EASTEND-RAVENSCRAG. George Doulias, Brian Lajeunesse, Gary Seib (compiler), Barbara, Beryl \& Peter Shourounis, John Sirounis

EASTEND-RAVENSCRAG. Tom Donald, Mike Gollop (compiler), Rick Jerema, John Mclntyre

ENDEAVOUR. Willie Haras

ENDEAVOUR. Barbara, David, Esther and Ronald Hooper

FEUDAL. Adam Schmidt, Guy Wapple

FORT QU'APPELLE. Nellie Hiebert, Ronald Hooper, Joe Kralkay, Lois Lamontagne, Vic Lamontagne, Alice Lang, Doreen Rowell, Lorne Rowell, Shawn Rowell, Lee-ann Sinclair, Dorothy Williams

FORT WALSH. Wayne Harris, Sheila Lamont, Don Renaud, Wayne Renaud (compiler) Guy Wapple

GARDINER DAM. Don and Wayne Renaud

GLAMIS-WISETON. Grev. Jones, David Nevin

GLASLYN-TURTLE LAKE. James Donovan

GRENFELL. Betty and John Hubbard

HAFFORD-BLAINE LAKE. Molly Denson, Ray Denson, Doug Leach, Lynn Oliphant, Bob Rafuse

HAWARDEN. Harold Kvinge

HUMBOLDT. Ed Brockmeyer, Clarence Saretsky, Isabel Saretsky

INDIAN HEAD. Cec and Betty Ashmore, Peter and Therese Barrett, Ernie and Hazel Buglass, Cam, Elizabeth and Shayne Davidson, Cindy Fell, Jim and Jean Howard, Gordon Howe, Rose McLaughlin, Lorne and Joan Scott, Ken and Mary Skinner (compiler)

ITUNA. John Petruka (compiler), Mary Brennan, Brian Ewasienko, Jeffrey Lozinski, Allan Petruka, Yvonne
KENASTON. P. Lawrence Beckie

KINDERSLEY. Jean Harris

KINDERSLEY. Gerry and Michael Essar KUTAWAGAN LAKE. (centered $19 \mathrm{~km}$ $\mathrm{N}$ of Semans) Duane Harding, Wayne Harris, Sheila Lamont

LANGHAM. Vi and Vic Harper

LAST MOUNTAIN LAKE (management unit and immediate area). Wayne Harris, Sheila Lamont

LEADER. Daisy D. Meyers

LUSELAND. Kerry and Kim Finley

MACDOWALL. John, Mary and Stan Shadick

MAIDSTONE BRIDGE $(34 \mathrm{~km} \mathrm{~N}$ of Maidstone). Wayne Harris, Sheila and Tom Lamont

MOOSE JAW. Alice and Carl Ellis, Doug Francis, Ruth Hilling, John Horton, Pat Kern, $\mathrm{Cy}$ and Leith Knight (compiler), Moray Lewis, Robert Pearson, Molly Ritchie, Sister Theresa and Jean Thomson

MOOSE MOUNTAIN PROVINCIAL PARK. Autumn Downey, Jim Hines

\section{NORTH BATTLEFORD. James \\ Donovan}

PIKE LAKE. Mr. and Mrs. Christensen, John, Mary and Stan Shadick

PIKE LAKE. Wendy Boehm, Bob Godwin, Ken Lumbis, Al, Bonnie and Edward Smith

PRINCE ALBERT. Wayne Harris, Sheila Lamont

PRINCE ALBERT NATIONAL PARK. Stuart Heard, David Henry, Suzanne Henry (compiler), Mike Jones, Virginia Plummer, Mervyn Syroteuk, Bruce Wilson, Pat Wilson, Charlie Zinkan, Marianne Zinkan

RAYMORE. Chas., Greta and Wayne Harris, Sheila Lamont

REGINA. Gary Anweiler, Jessie Bailey, Margaret Belcher, Tom Beveridge, Howard Biliak, Frank Brazier, K'. Costain, Betty Cruikshank, Robyn Donison, Autumn Downey, J. G. Ducreux, Mr. and Mrs. George Forsythe, Wayne Gemmell, Jim Hines (compiler), Roger Howard, Jim and Shirley Jowsey, Ernie and Elizabeth Kassian, Darlene Kauk, Greg Kraetzig, Bob Kreba, Tony Lang, George Ledingham, Rita Leflar, Bob Luterbach, Wayne Lynch, Mrs. G. McKay, Sally Moss, Brad'Muir, Eric Roberts, 
Joe Roberts, Diane and Allan Smith, C Wilhelm, Pierre Wilhelm

ROSETOWN. Don and Wayne Renaud

ROUND LAKE. Doug Francis, Bill Middleton

\section{SALTCOATS. Robert Barnhart}

SASKATOON. Juhachi Asai, Bob, Garth, Joan and Joyce Besant, Ron Bobowski, Andre Bouthillette, Ron and Muriel Bremner, Nigel Caulkett, Ian Creurer, Maureen DuWors, Alan, Arthur, Hartley and Lawrence Fredeen, Geof and Muriel Galloway, Bob Godwin, Bernie and Madeleine Collop, Bruce and|John Hanbidge, C. J. David, Donald, Mary and Stuart Houston, Andrew and Charles Hope, Sandra and Tony Johns, Don and Jo McRobbie, David Miller, Betty Mundy, Pat O'Neil, John Polson, John and Stan Shadick, Jim Slimmon, Alan and Ed Smith

SCOTT. Don and Wayne Renaud, Guy Wapple

SKULL CREEK. Jim Bennetto

SKULL CREEK. Jim Bennetto, Sybella and Austin Drever, Phyllis Flaig, Betty and Patty Mann, Ray and Michelle Schuler

SOMME. David Black, Donald and Ronald Hooper

SPIRIT LAKE. Bill and Joyce Anaka

SPRING VALLEY. Adeline and Allan Bogdan, Flossie and Nick Bogdan

SQUAW RAPIDS-CUT BEAVER. John Comer, Terry Klassen

TOGO. Phil and Jean Hern, Vic and Olga Hilderman, Walter and Ethel Krupp, Cusie and Wanda May (compiler), Dick and Mary Smith, Howard and Donelda Wilson, Orville and Elaine Wilson

VALLEY CENTRE. Guy Wapple

WHITE BEAR. Bill Fox, Tim Fox, Laine Jordheim, Sig Jordheim, Mark Lowe, Doug Stepples, Doug Strom

WHITEBEECH. Lindsay Wotherspoon WOLSELEY. J. Donald Hayward, Doreen Hayward

YORKTON. Gerry and Michael Essar

Table 2. CHRISTMAS COUNT METHODS OF OBSERVATION, WEATHER AND TOTAL BIRDS

\begin{tabular}{|c|c|c|c|c|c|c|c|c|c|c|c|c|c|}
\hline \multirow[b]{2}{*}{ Place } & \multicolumn{2}{|c|}{ Car } & \multicolumn{2}{|c|}{ Foot } & \multicolumn{2}{|c|}{ Other } & & \multirow[t]{2}{*}{ Temp ${ }^{\circ} \mathrm{C}$} & \multicolumn{2}{|c|}{ Wind } & \multirow{2}{*}{$\begin{array}{l}\text { Snow } \\
\text { Cover } \\
\mathrm{CM}\end{array}$} & \multirow[t]{2}{*}{ Sky } & \multirow{2}{*}{$\begin{array}{c}\text { Count } \\
\text { Day } \\
\text { Number } \\
\text { Individual }\end{array}$} \\
\hline & $K M$ & $\mathrm{HR}$ & KM & $\mathrm{HR}$ & $K M$ & HR & & & DIR & $\mathrm{KM} / \mathrm{H}$ & & & \\
\hline Bangor & 14 & & & & & & & -20 & & 0 & & & 59 \\
\hline Battleford - & & & & & & & & & & & & & \\
\hline North Battleford & 56 & 3 & & & & & & -20 & & light & 15 & & 2561 \\
\hline Beaver Creek & & & & & on $\mathrm{s}$ & kis & & -6 & & & & sunny & 43 \\
\hline Besnard Lake & 24 & $1 / 2$ & 3 & $1 \frac{1}{2}$ & & & & & & & & & 51 \\
\hline Biggar & 159 & 9 & 16 & 8 & $\begin{array}{c}\text { snowm } \\
45\end{array}$ & $\begin{array}{c}\text { obile } \\
6\end{array}$ & & -12 to -16 & WNW & $8-24$ & $10-40$ & $\begin{array}{l}\text { mostly } \\
\text { cloudy }\end{array}$ & 2932 \\
\hline Big Gully Creek & 56 & 3 & 5 & 2 & \begin{tabular}{|l|} 
on s \\
8 \\
\end{tabular} & $\begin{array}{c}\text { is } \\
2 \\
\end{array}$ & & -16 to -10 & NW & $20-25$ & 45 & overcast & 586 \\
\hline Broadview & 147 & 3 & & & \begin{tabular}{|c} 
on snow \\
7 \\
\end{tabular} & $\begin{array}{c}\text { shoes } \\
4\end{array}$ & & -20 & & 0 & & $\begin{array}{l}\text { mostly } \\
\text { cloudy }\end{array}$ & 810 \\
\hline Candle Lake & 56 & 2 & 16 & 6 & & & & -31 to -18 & NW & $15-20$ & 25 & overcast & 141 \\
\hline Clarkboro & & & & & $\begin{array}{c}\text { on } 5 \\
5 \\
\end{array}$ & & & -9 & NWr & modera & & cloudy & 29 \\
\hline Cold River & 24 & 1 & & & on snow & $\begin{array}{c}\text { shoes } \\
3\end{array}$ & & -15 & NW & 25 & 40 & $\begin{array}{l}\text { overcast } \\
\text { light snow }\end{array}$ & 106 \\
\hline Dalmeny & & & & & $\begin{array}{c}\text { on } 5 \\
27\end{array}$ & & & -12 to -14 & $S$ & light & & $\begin{array}{c}\text { Overcast } \\
\text { light snow }\end{array}$ & 274 \\
\hline Dilke & 26 & 2 & 2 & $1 \frac{1}{2}$ & $\begin{array}{l}\text { on } s \\
3 \\
\end{array}$ & & & -8 to -10 & NW & 0 to lis & ight & & 231 \\
\hline Duck Lake & 32 & & & & $\begin{array}{l}\text { on } 5 \\
6 \\
\end{array}$ & \begin{tabular}{|l|} 
is \\
$21 / 2$
\end{tabular} & & & & & & & 82 \\
\hline Duperow - & 145 & $41 / 2$ & 10 & $41 / 2$ & snowm & obile & & -10 to -14 & $w$ & $8-24$ & $15-45$ & clear & 2205 \\
\hline Ruthilda & & & & & 7 & $1 / 2$ & & & & & & & \\
\hline Eastend - & 64 & $21 / 2$ & 5 & 2 & & & & -10 & & & & & 492 \\
\hline Ravenscrag & & & & & & & & & & & & & \\
\hline $\begin{array}{l}\text { Eastend - } \\
\text { Ravenscrag }\end{array}$ & 166 & & 16 & & & & & -16 to -26 & WNW & 24 & & $\begin{array}{l}\text { mostly } \\
\text { clear }\end{array}$ & 875 \\
\hline Endeavour & & & & & $\begin{array}{c}\text { arol } \\
\text { farm }\end{array}$ & $\begin{array}{l}\text { ind } \\
\text { yard }\end{array}$ & & -34 to -8 & & 0 & 36 & clear & 585 \\
\hline Endeavour & 48 & 1 & & & & & & -18 & & 0 & & sunny & 61 \\
\hline Feudal & 93 & 4 & 4 & $1 \frac{1 / 2}{2}$ & & & & -8 & $N$ & $35-45$ & $25-40$ & $\begin{array}{l}\text { mostly } \\
\text { clear }\end{array}$ & 1493 \\
\hline Fort Qu'Appelle & 96 & 4 & & & & & & -18 & & 0 & 46 & cloudy & 481 \\
\hline For: Walsh & 81 & 3 & 42 & 24 & & & & -8 to -10 & NW & $15-20$ & $5-45$ & $\begin{array}{l}\text { overcast } \\
\text { some snow }\end{array}$ & 3129 \\
\hline
\end{tabular}


Table 2. CHRISTMAS COUNT METHODS OF OBSERVATION, WEATHER AND TOTAL BIRDS

\begin{tabular}{|c|c|c|c|c|c|c|c|c|c|c|c|c|c|}
\hline \multirow[b]{2}{*}{ Place } & \multicolumn{2}{|c|}{ Car } & \multicolumn{2}{|c|}{ Foot } & \multicolumn{2}{|c|}{ Other } & \multirow{2}{*}{$\mathrm{e}_{\mathrm{d}_{\mathrm{e}}}$} & \multirow{2}{*}{ Temp ${ }^{\circ} \mathrm{C}$} & \multicolumn{2}{|c|}{ Wind } & \multirow{2}{*}{$\begin{array}{l}\text { Snow } \\
\text { Cover } \\
\mathrm{CM}\end{array}$} & \multirow[t]{2}{*}{ Sky } & \multirow[t]{2}{*}{$\begin{array}{c}\text { Count } \\
\text { Day } \\
\text { Number } \\
\text { Individuals }\end{array}$} \\
\hline & $K M$ & $H R$ & $K M$ & $H R$ & KM & HR & & & DIR & $K M / H$ & & & \\
\hline Gardiner Dam & 40 & 2 & 7 & 3 & & & & -11 to -14 & W & $8-16$ & 40 & overcast & 797 \\
\hline Glamis - & 51 & 2 & 2 & 1 & & & & -24 to -20 & & 0 & & partly & 147 \\
\hline Wiseton & & & & & & & & & & & & sunny & \\
\hline Claslyn - & 24 & 1 & & & \multicolumn{2}{|c|}{ snowshoes } & & -15 & $W$ & $15-20$ & \multicolumn{2}{|c|}{ overcast a.m. } & 327 \\
\hline Turtle Lake & & & & & 8 & 4 & & & & & \multicolumn{2}{|r|}{ sunny p.m. } & \\
\hline Grenfell & 10 & & & & \multicolumn{2}{|c|}{$\begin{array}{l}\text { on skis } \\
3\end{array}$} & & -23 & $\bar{W}$ & 16 & 36 & & 110 \\
\hline Hafford - & 117 & $31 / 2$ & 3 & 1 & \multicolumn{2}{|c|}{ on skies } & & -28 to -20 & & & & & 508 \\
\hline Blaine Lake & & & & & 5 & 2 & & & & & & & \\
\hline Hawarden & & & & & & & & & & & & & 2 \\
\hline Humboldt & 32 & & & & $9^{\text {on }}$ & $\mathrm{k}$ is & & -10 & & 0 & & sunny & 78 \\
\hline Indian Head & 16 & 1 & 2 & 1 & by tr & actor & & -10 & & light & 55 & overcast & 1076 \\
\hline Ituna & 35 & & & & & & & & & & & & 210 \\
\hline Kenaston & & 5 & & & & & & & SW & Light & 25 & $\begin{array}{c}\text { Light } \\
\text { Overcast }\end{array}$ & 67 \\
\hline Kindersley & 14 & & 2 & & & & & -18 & & 0 & 20 & Sunny & 49 \\
\hline Kindersley & & & & & $\begin{array}{l}\text { Back } \\
\text { In T }\end{array}$ & $\begin{array}{l}\text { Yard } \\
\text { own }\end{array}$ & & & & & & & 126 \\
\hline Kutawagan Lake & 48 & 2 & & & $\begin{array}{c}\text { Snow } \\
6\end{array}$ & $\begin{array}{c}\text { shoes } \\
3\end{array}$ & & -20 to -18 & SE & 10 & 40 & Overcast & 164 \\
\hline Langham & & & & & Farm & Yard & & & & & & & 70 \\
\hline Last Mountain Lake & 128 & 5 & 3 & 2 & & & & -24 to -18 & NW & $15-30$ & 40 & Sunny & 1710 \\
\hline Leader & & & & 4 & & & & -4 & & 0 & 40 & Clear & 112 \\
\hline Luseland & 100 & 3 & 10 & 4 & & & & -15 & NW & 5 & $30-35$ & $\begin{array}{l}\text { Light } \\
\text { Overcast }\end{array}$ & 858 \\
\hline MacDowall & 59 & 5 & & & & & & $\begin{array}{r}-19 \\
\end{array}$ & & & & Clear & 216 \\
\hline Maidstone Bridge & 88 & 4 & 6 & 4 & & & & -16 to -9 & NW & 12 & 45 & Overcast & 960 \\
\hline Moose law & 180 & & 11 & & & & & $\begin{array}{r}-10 \\
\end{array}$ & & Light & 15 & Sunny & 1591 \\
\hline Moose Mountain & 78 & 6 & 1 & $1 / 2$ & $\begin{array}{c}\text { On } \\
13\end{array}$ & $\begin{array}{c}\text { Skis } \\
4\end{array}$ & & -30 to -25 & & 0 to 10 & $025-50$ & $\begin{array}{l}\text { Cloudy } \\
\text { Clearing }\end{array}$ & 111 \\
\hline North Battleford & & & 8 & 5 & & & & -15 & & Light & & & 360 \\
\hline Pike Lake & 40 & 3 & & & & & $5 \mathrm{Hr}$ & & & & & & 2051 \\
\hline Pike Lake & 24 & $11 / 2$ & 3 & $1 \frac{1}{2}$ & On & Skis & & & & & & & 498 \\
\hline Prince Albert & 136 & 4 & 8 & 3 & & & & -30 to -24 & NW & 15 & 25 & $\begin{array}{l}\text { Mostlv } \\
\text { Clear }\end{array}$ & 1769 \\
\hline Prince Albert & 90 & & 43 & & & & & -23 to -18 & NNW & $5-10$ & 25 & Overcast & -86 \\
\hline Vational Park & & & & & & & & & & & & Clearing & \\
\hline Raymore & 176 & 7 & 3 & 1 & $\begin{array}{r}\text { Ski } \\
\text { Snow } \\
7\end{array}$ & $\begin{array}{l}\text { st } \\
\text { shoes } \\
3\end{array}$ & & -22 to -10 & $\overline{S E}$ & $0-10$ & 50 & $\begin{array}{l}\text { Fog } \\
\text { Brief Sun }\end{array}$ & 962 \\
\hline Regina & 544 & 39 & 61 & 27 & & & & -11 to -8 & NW & $25-50$ & 25-50 & $\begin{array}{c}\text { Overcast } \\
\text { Light Snow }\end{array}$ & 6406 \\
\hline Rosetown & 48 & 2 & 2 & 1 & & & & -21 & NW & $8-24$ & 40 & Clear & 282 \\
\hline Round Lake & 80 & 4 & 3 & 3 & & & & & & & & & 422 \\
\hline Saltcoats & 66 & $31 / 2$ & & & & & 2 & -15 & & 0 & & Overcast & 269 \\
\hline Saskatoon. & 265 & $42 \%$ & 36 & 33 & $\begin{array}{l}\text { On } \\
15\end{array}$ & $\begin{array}{r}\text { Skis } \\
13 \\
\end{array}$ & $16 \mathrm{Hr}$. & -26 to -18 & SW & $8-15$ & 16 & Overcast & 11,843 \\
\hline Scott & 121 & $51 / 2$ & 7 & $41 / 2$ & & & & -6 to -11 & $w$ & $8-40$ & 40 & Overcast & 1,092 \\
\hline Skull Creek & & & & & & & & & & & & & 993 \\
\hline Skull Creek & & & & & $\begin{array}{c}\text { Snow } \\
+\end{array}$ & $\begin{array}{l}\text { mobile } \\
\text { ikis }\end{array}$ & & -14 & & & & Light & 933 \\
\hline Somme & 104 & 2 & 10 & 3 & & & & -12 & & Modera & te 30 & Overcast & 405 \\
\hline Spirit Lake & 88 & $41 / 2$ & 3 & 2 & & & & -12 to -10 & & $\begin{array}{l}\text { Calm } \\
\text { to Ligh }\end{array}$ & 35 & Overcast & 336 \\
\hline Spring Valley & 58 & $11 / 2$ & & & Aroun & $\begin{array}{c}d \text { Yard } \\
1\end{array}$ & & -20 & $w$ & Light & 60 & Cloudy & 558 \\
\hline Squaw Rapids - & 80 & 2 & 2 & 2 & & & & -15 to -20 & & Light & & Overcast & 278 \\
\hline Cut Beaver & & & & & & & & & & & & & \\
\hline Togo & & & & & & & 7 & & & & & & 461 \\
\hline Valley_entre & 97 & 4 & 5 & $21 / 2$ & & & & -8 to -14 & W & $5-30$ & $10-30$ & overcast & 1839 \\
\hline Waseca. & & & & & $\begin{array}{r}\text { farmst } \\
\text { ma }\end{array}$ & $\begin{array}{l}\text { ead \& } \\
\text { rsh }\end{array}$ & & & & light & & $\begin{array}{l}\text { partly } \\
\text { sunny }\end{array}$ & 92 \\
\hline Webb & 112 & 4 & 5 & $11 / 2$ & & & & -15 & & light & & $\begin{array}{l}\text { mostly } \\
\text { sunny }\end{array}$ & 107 \\
\hline White Bear & & & & & & & & -12 & NNE & 10 & & snowing & 47 \\
\hline Whitebeech & 3 & 2 & & & & & & & & & & & 17 \\
\hline Wolseley & & & & & & & & -18 & & 0 & 39 & & $\frac{374}{126}$ \\
\hline Yorkton & 128 & & & & & & & & & & & snow & 126 \\
\hline
\end{tabular}


Table 3. BIRDS RECORDED FROM MORE THAN ONE LOCALITY ON CO DAY AND IN COUNT PERIOD + )

\begin{tabular}{|c|c|c|c|c|c|c|c|c|c|c|c|c|c|c|c|c|}
\hline & 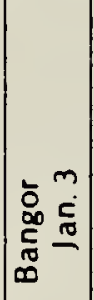 & 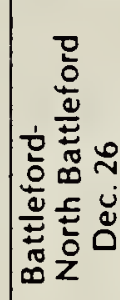 & 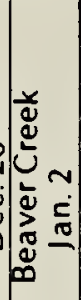 & 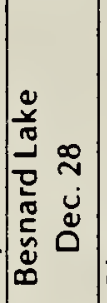 & 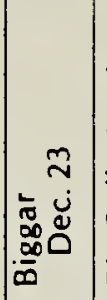 & 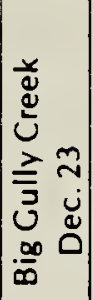 & 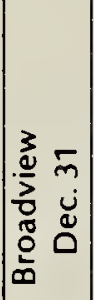 & 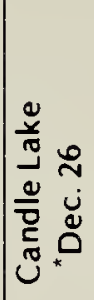 & 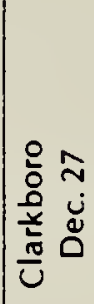 & 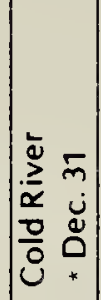 & 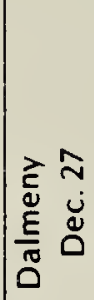 & 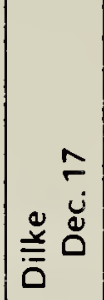 & $\mid$\begin{tabular}{|c} 
\\
\\
0
\end{tabular} & 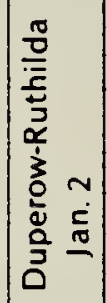 & 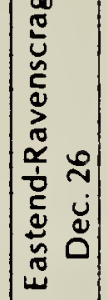 & 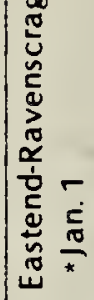 \\
\hline \multicolumn{17}{|l|}{ Canada Coose } \\
\hline Mallard & & & & & & & & & & 13 & & & & & 79 & \\
\hline \multicolumn{17}{|l|}{ Redhead } \\
\hline Common Coldeneye & & & & & & & & & & 3 & & & & & & \\
\hline Common Merganser & & & & & & & & & & 2 & & & & & & \\
\hline Coshawk & & & & & & & & 1 & & & 1 & & & & & 1 \\
\hline Colden Eagle & & & & & 1 & & 1 & & & & & & & +1 & +1 & 1 \\
\hline Bald Eagle & & & & & +1 & & & & & 1 & & & & & & \\
\hline \multicolumn{17}{|l|}{ Gyrifalcon } \\
\hline Prairie Falcon & & & & & & & & & & & & & & & 2 & 1 \\
\hline Merlin & & & & & 1 & & & & & & & & & & & $T$ \\
\hline Ruffed Crouse & 1 & & & & & & & 8 & & 1 & & & 1 & & & \\
\hline Sharp-Tailed Grouse & & & & & 99 & 1 & 43 & & & & 10 & +14 & & 10 & & 1 \\
\hline Sage Crouse & & & & & & & & & & & & & & & 2 & +35 \\
\hline Ring-Necked Pheasant & & & & & & & 1 & & & & & 1 & & & 1 & +7 \\
\hline Cray Partridge & & & & & 27 & +6 & 5 & & & & 75 & 12 & & 80 & 4 & 25 \\
\hline Rock Dove & & 34 & & & 63 & +1 & 13 & & & & & 12 & & 60 & +11 & 22 \\
\hline Great Horned Owl & & 1 & & & 3 & & +1 & 1 & & & & & & 1 & & 1 \\
\hline Snowy Owl & & & & & +1 & 1 & & & & & 1 & & & 2 & & 1 \\
\hline Hawk Owl & & 2 & & & & & & & & & & & & & & \\
\hline Great Gray Owl & & & & 1 & & & & 1 & & & & & & & & \\
\hline \multicolumn{17}{|l|}{ Saw-whet Owl } \\
\hline \multicolumn{17}{|l|}{ Common Flicker } \\
\hline Pileated Woodpecker & & & & & & 1 & & & & & & & & & & \\
\hline Hairy Woodpecker & 2 & & & & 1 & 1 & 3 & 1 & & & 1 & 1 & & & +1 & 2 \\
\hline Downy Woodpecker & 1 & 1 & 1 & & 1 & 2 & +1 & 2 & 1 & & & & & +1 & 2 & 1 \\
\hline \multicolumn{17}{|l|}{ Bl-Bk 3-T Woodpecker } \\
\hline North 3-T Woodpecker & & & & +1 & & & & & & & & & & & & \\
\hline Horned Lark & & & & & 50 & & & & & & & 2 & & & 68 & 6 \\
\hline Graylay & & & & & & & & 12 & & 2 & & & 4 & & & \\
\hline Blue Jay & & & & & +1 & 4 & $T$ & 1 & & & & & 1 & & & \\
\hline Black-Billed Magpie & $\frac{1}{1}$ & 6 & $2^{1}$ & & 160 & 21 & 61 & 15 & 1 & 1 & 6 & 1 & & 148 & 60 & 129 \\
\hline Common Raven & & & & 50 & & & & 57 & & 5 & +1 & & 3 & & & \\
\hline Black-Cap Chickadee & 4 & & 15 & +1 & 6 & 4 & 16 & 8 & 2 & & 1 & & 7 & 9 & 7 & 52 \\
\hline Boreal Chickadee & & & & & & & & 11 & & & & & 3 & & & \\
\hline \multicolumn{17}{|l|}{ Wh-Breast Nuthatch } \\
\hline \multicolumn{17}{|l|}{ Red-Breast Nuthatch } \\
\hline Brown Creeper & & & & & & & & & & & & & 1 & & & \\
\hline American Robin & & & & & & & & & & & & & & & +2 & 2 \\
\hline Bohemian Waxwing & & 2500 & & & 162 & & $+\overline{36}$ & & & & +30 & & & 2 & 225 & 30 \\
\hline \multicolumn{17}{|l|}{ Cedar Waxwing } \\
\hline Northern Shrike & & & & & & & & & & & 1 & & & & & \\
\hline Starling & & & & & 5 & & 20 & & & & & & & 6 & +1 & 44 \\
\hline House Sparrow & & 11 & & & 1218 & 30 & 30 & & & & 40 & 100 & & 1586 & 64 & 330 \\
\hline Rusty Blackbird & & & & & & & & & & & & & & & & 1 \\
\hline Evening Grosbeak & & 4 & & & & +2 & 5 & & & & & & & & & \\
\hline Pine Grosbeak & & 2 & & & 19 & 13 & 35 & 11 & & & 19 & +1 & 2 & 36 & 20 & 38 \\
\hline Gray-Cr Rosy Finch & & & & & & & & & & & & & & & & +6 \\
\hline Hoary Redpoll & & & & & 3 & 3 & +5 & & & & & & & 2 & & \\
\hline Common Redpoll & & & 25 & +60 & 595 & 142 & 93 & 10 & & 53 & 44 & 2 & 60 & 204 & 37 & 185 \\
\hline \multicolumn{17}{|l|}{ Dark-Eyed Junco } \\
\hline \multicolumn{17}{|l|}{ Tree Sparrow } \\
\hline \multicolumn{17}{|l|}{ Lapland Longspur } \\
\hline Snow Bunting & 50 & & & & 518 & 363 & 483 & 1 & 25 & 249 & 150 & 100 & & 55 & & 1 \\
\hline Count Day Species & 6 & 9 & 4 & 2 & 18 & 13 & 15 & 16 & 4 & 11 & 11 & 9 & 9 & 15 & 12 & 22 \\
\hline Count Period Species & 6 & 9 & 4 & 5 & 21 & 16 & 19 & 16 & 4 & 11 & 14 & 11 & 9 & 17 & 18 & 27 \\
\hline
\end{tabular}

* See also list of species recorded from 1 location only.

+ Species seen during count period (Dec. 17, 1977-Jan. 2, 1978) but not on count day. 


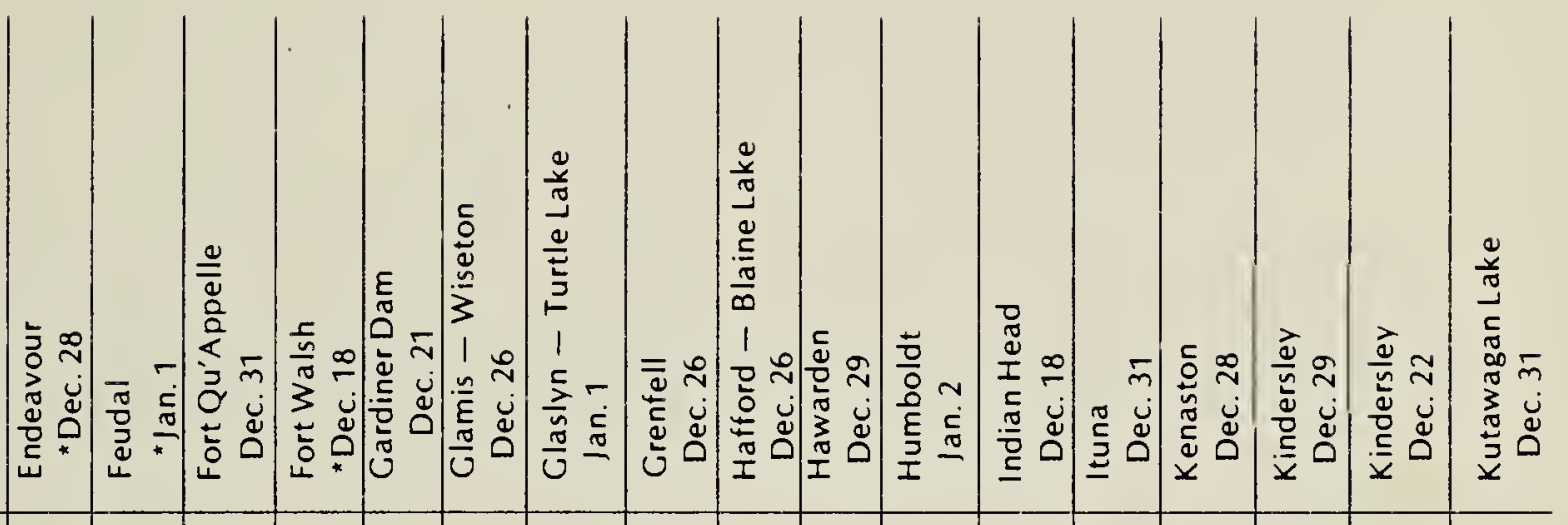

Canada Coose

Mallard

Redhead

Common Coldeneye

Common Merganser

Coshawk

Colden Eagle

Bald Eagle

Cyrfalcon

Prairie Falcon

Merlin

Ruffed Crouse

Sharp-Tailed Crouse

Sage C rouse

Ring-Necked Pheasant

Cray Partridge

Rock Dove

Great Horned Ow

Snowy Owl

Hawk Owl

Creat Cray Owl

Saw-Whet Owl

Common Flicker

Pileated Woodpecker

Hairy Woodpecker

Downy Woodpecker

Bl-Bk 3-TWoodpecker

North 3-T Woodpecker

Horned Lark

Cray Jay

Blue Jay

Black-Billed Magpie

Common Raven

Black-Cap Chickadee

Boreal Chickadee

Wh-Breast Nuthatch

Red-Breast Nuthatch

Brown Creeper

American Robin

Bohemian Waxwing

Cedar Waxwing

Northern Shrike

Starling.

House Sparrow

Rusty Blackbird

Evening Crosbeak

\begin{tabular}{|r|r|}
+2 & 130 \\
\hline
\end{tabular}

\begin{tabular}{|c|c|c|c|c|}
\hline & T & +2 & & 130 \\
\hline & & & & \\
\hline & & & & 15 \\
\hline & & & & 3 \\
\hline & & & 1 & \\
\hline & & & 5 & \\
\hline & & & 4 & 1 \\
\hline
\end{tabular}

ine Grosbeak

Gray-Cr Rosy Finch

Hoary Redpoll

Common Redpol

Jark-Eyed Junco

ree Sparrow

apland Longspur

now Bunting

\begin{tabular}{|r|r|r|r|r}
\hline & & & & \\
\hline & & 1 & 2 & \\
\hline & & 4 & 21 & \\
\hline & & & 12 & \\
\hline & 9 & 5 & 5 & 12 \\
\hline & 3 & 30 & 3 & 1 \\
\hline & +1 & & 1 & 1 \\
\hline
\end{tabular}

$+$

\begin{tabular}{|l|l|l|l|l|l|}
\hline & & & & & \\
\hline
\end{tabular}

$+$

\begin{tabular}{ll|l|l|l}
+1 & & \\
\hline & & & \\
\hline
\end{tabular}

\begin{tabular}{rr|r|r|r|r|r|r|r|r|r|r|r} 
& & & & & & & & & & & & \\
\hline & 2 & & & 19 & & 5 & & 40 & 5 & & & 2 \\
\hline & & & & & & & & & & & & \\
\hline 2 & 20 & & +9 & & & +5 & +10 & +2 & 21 & & & \\
\hline
\end{tabular}

\begin{tabular}{rrrr|r|r|r|r|r|r|r|r|r|r|r|r} 
& & & & & & & & & & & & & & & \\
\hline & 2 & 2 & 1 & & & 1 & 2 & & 1 & 5 & & & & & \\
\hline & & 4 & & & 1 & +1 & 1 & & & 9 & & & & & 1 \\
\hline & & 5 & & & & & & & & & & & & & \\
\hline
\end{tabular}

\begin{tabular}{r|r|r|r|r|r|r|r|r|r|r|r}
\hline 2 & 1 & & & & +1 & 1 & & & 1 & & +1 \\
\hline & & & & & & & & & & & \\
\hline
\end{tabular}

\begin{tabular}{rr|r|r|r|r|r|r|r|r|r|r|r|r|r|r|r|r}
\hline 2 & 46 & +1 & 90 & & & & +1 & & & & & & & & & 2 \\
\hline 1 & & 1 & 1 & & & & & & & +1 & +3 & & & & & \\
\hline
\end{tabular}




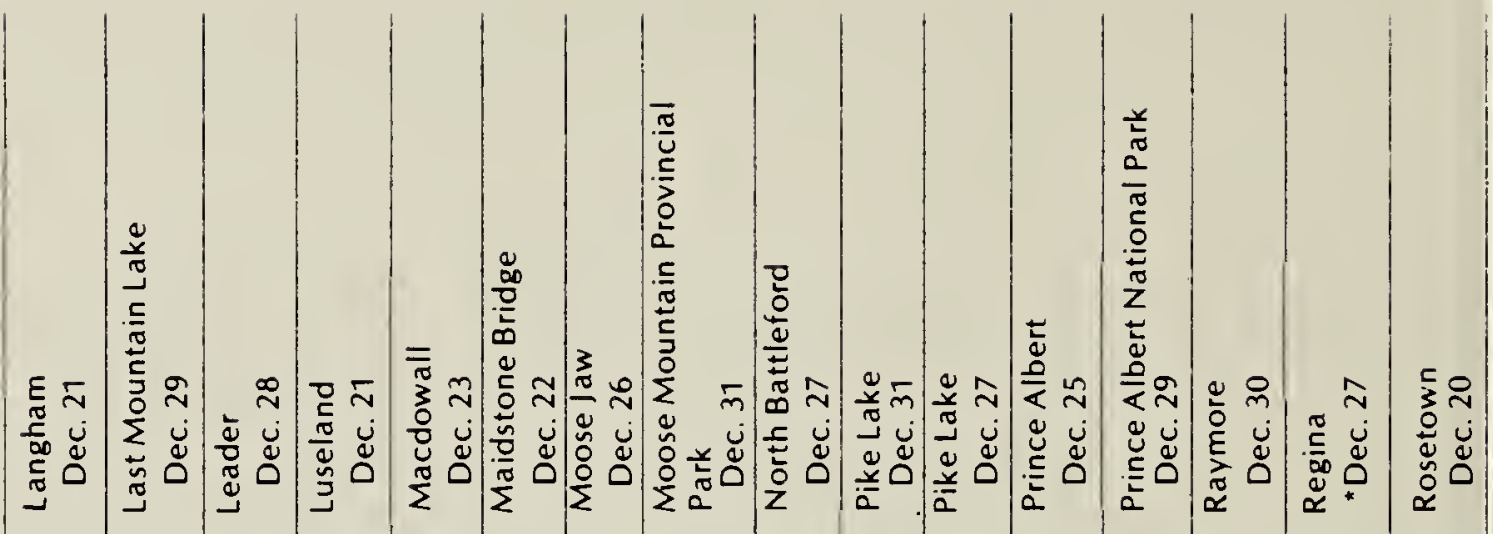

Canada Coose

Mallard

Redhead

Common Goldeneye

Common Merganser

Coshawk

Golden Eagle

Bald Eagle

Gyrfalcon

Prairie Falcon

Merlin

Ruffed Grouse

Sharp-Tailed Grouse

Sage Grouse

Ring-Necked Pheasant

Gray Partridge

Rock Dove

Great Horned Owl

Snowy Owl

Hawk Owl.

Great Gray Owl

Saw-Whet Owl

Common Flicker

Pileated Woodpecker

Hairy Woodpecker

Downy Woodpecker

Bl-Bk 3-T Woodpecker

North 3-T Woodpecker

Horned Lark

Gray Jay

Blue Jay

Black-Billed Magpie

Common Raven

Black-Cap Chickadee

Boreal Chickadee

Wh-Breast Nuthatch

Red-Breast Nuthatch

Brown Creeper

American Robin

Bohemian Waxwing

CedarWaxwing

Northern Shrike

Starling

House Sparrow

Rusty Blackbird

Evening Crosbeak

Pine Grosbeak

Gray-Cr Rosy Finch

Hoary Redpoll

Common Redpoll

Dark-Eyed Junco

Tree Sparrow

Lapland Longspur

Snow Bunting

\begin{tabular}{r|r|}
\hline & $\frac{1174}{304}$ \\
\hline & 1 \\
\hline & 2
\end{tabular}




\begin{tabular}{|c|c|c|c|c|c|c|c|c|c|c|c|c|c|c|c|c|c|c|c|}
\hline & 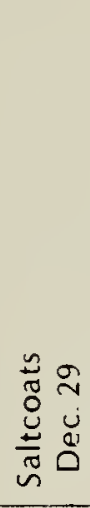 & 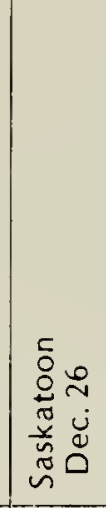 & 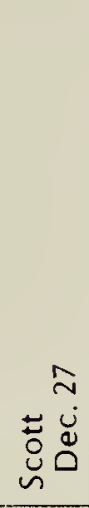 & 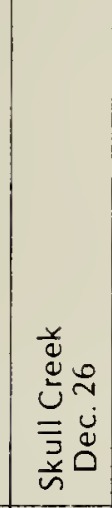 & 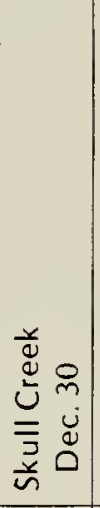 & 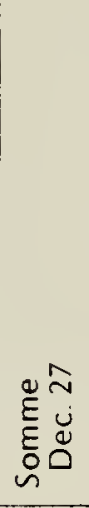 & 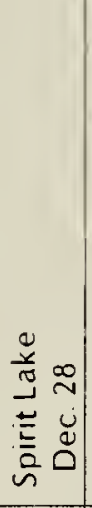 & 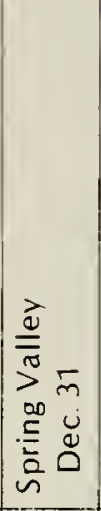 & 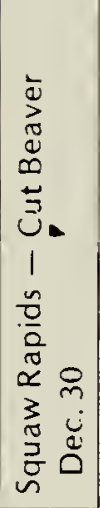 & 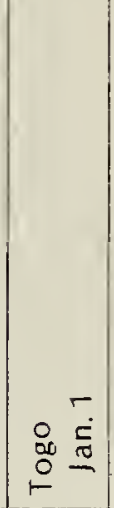 & 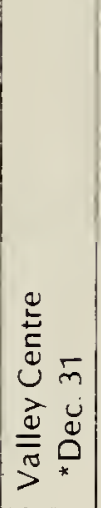 & 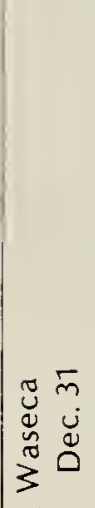 & $\begin{array}{ll} & 0 \\
0 & \dot{v} \\
0 & 0 \\
ن & 0 \\
3 & 0\end{array}$ & 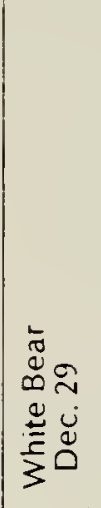 & 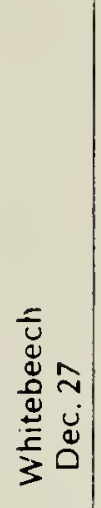 & 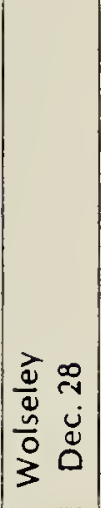 & 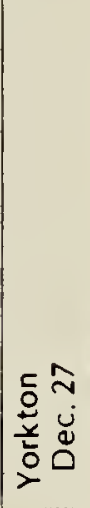 & 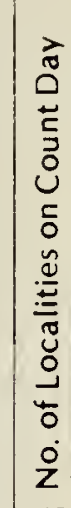 & 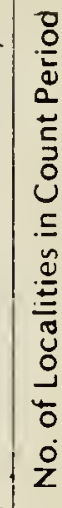 \\
\hline Coose & & & & & & & & +1 & & & & & & & & & & 1 & 2 \\
\hline 1 & & 14 & & & & & & & 22 & & & & & & & & & 6 & 8 \\
\hline d & & 2 & & & & & & & & & & & & & & & & 2 & 2 \\
\hline in Coldeneye & & 134 & & & & & & & 45 & & & & & & & & & 5 & 5 \\
\hline on Merganser & & 1 & & & & & & & 4 & & & & & & & & & 4 & 4 \\
\hline k & & 1 & & & & 1 & +1 & & & & & & & & & & & 8 & 9 \\
\hline Eagle & & & 1 & 1 & & & & +1 & 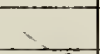 & & 1 & & & 1 & & & & 10 & 15 \\
\hline gle & & & & 2 & & & & & 2 & & & & & & & & & 7 & 8 \\
\hline on & & & & & & & & & & & & & & & & & & 0 & 2 \\
\hline Falcon & & & & 1 & & & & & & & & & & & & & & 5 & 5 \\
\hline & & 6 & & & & & 1 & & & & & & & 1 & & & & 8 & 8 \\
\hline Grouse & & 4 & & & & 1 & 1 & & & 1 & & 9 & & & & 5 & & 19 & 22 \\
\hline ailed Grouse & 15 & 83 & 2 & 18 & 71 & 16 & 6 & 26 & & 5 & 6 & & & & & 6 & & 34 & 36 \\
\hline ouse & & & & & & & & & & & & & & & & & & 1 & 3 \\
\hline cked Pheasant & & 35 & & 9 & 11 & & & & & & & & & 1 & & & & 13 & 14 \\
\hline rtridge & & 58 & 14 & 23 & 53 & & 14 & 83 & & 10 & 104 & & 34 & 14 & & 8 & & 31 & 39 \\
\hline ove & & 981 & 39 & 7 & 13 & +4 & +2 & 26 & & & 3 & & 8 & & & 25 & & 34 & 39 \\
\hline orned Owl & 2 & 7 & 1 & 1 & & & +1 & +1 & 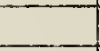 & & 2 & +1 & & & & +1 & & 18 & 29 \\
\hline Dwl & & 1 & 2 & & & & & +1 & & & & & 1 & & & & & 15 & 20 \\
\hline$w 1$ & & 1 & & & & & & & 1 & & & & & & & & & 3 & 3 \\
\hline ray Owl & & & & & & & & & & & & & & & & & & 3 & 3 \\
\hline iet Owl & & +1 & & & & & & & & & & & & & & & & 2 & 4 \\
\hline n Flicker & & 1 & & & & & & & & & & & & & & & & 2 & 2 \\
\hline Woodpecker & & & & & & & & & & 1 & & & & & & & & 2 & 2 \\
\hline oodpecker & 4 & 8 & 1 & & 2 & 5 & 7 & & & 7 & & 3 & & 1 & 3 & 2 & & 37 & 39 \\
\hline Woodpecker & 5 & 22 & & 4 & 4 & & 9 & & & 5 & & 2 & & & 2 & 1 & & 35 & 39 \\
\hline TWoodpecker & & & & & & 3 & & & & & & & & & & & & 2 & 2 \\
\hline I Woodpecker & & & & & & 8 & & & & & & & & & & & & 3 & 5 \\
\hline Lark & & & 3 & +400 & 7 & & & 8 & & & 15 & & 40 & & & & & 15 & 19 \\
\hline 1 & & +1 & & & & 3 & 1 & & 1 & 4 & & & & & 3 & & & 13 & 17 \\
\hline & 4 & 35 & & & & 9 & 1 & & & 66 & & 3 & & & & & & 25 & 28 \\
\hline Hled Magpie & 4 & 329 & 49 & 16 & 26 & 18 & 22 & 7 & 9 & +4 & 79 & 2 & 2 & 2 & 1 & 6 & 5 & 63 & 64 \\
\hline Raven & & & & & & 38 & +1 & & 26 & 2 & & & & & 1 & & & 15 & 17 \\
\hline p Chickadee & 26 & 131 & 8 & 6 & 21 & 6 & 42 & & 3 & 39 & 3 & 4 & & & 7 & 4 & & 53 & 55 \\
\hline hickadee & & & & & & 3 & & & & 2 & & & & & & & & 6 & 6 \\
\hline st Nuthatch & 1 & 3 & & & & & 2 & & & +1 & & & & & & & & 11 & 14 \\
\hline ast Nuthatch & & 2 & & & & & & & & & & +1 & & & & & & 1 & 2 \\
\hline reeper & & & & & & & & & & & & & & & & & & 4 & 4 \\
\hline n Robin & & 19 & & 1 & & & & & & & & & & +1 & & & & 9 & 11 \\
\hline n Waxwing & 2 & 4980 & 16 & 163 & 353 & & +1 & +18 & & & 188 & & 10 & 25 & & +1 & 25 & 31 & 40 \\
\hline axwing & & 3 & & & & & & & & & & & +3 & & & & & 2 & 4 \\
\hline \multirow[t]{2}{*}{ Shrike } & & 4 & & & & & & & +1 & & & & & & & & & 7 & 11 \\
\hline & & 96 & & & & & & 6 & & & & & +1 & & & & & 14 & 17 \\
\hline parrow & 50 & 1945 & 274 & 185 & 177 & 62 & 208 & 360 & & 49 & 925 & & 12 & & & 100 & & 50 & 52 \\
\hline ackbird & & & & & & & & & & & & & & & & & & 2 & 2 \\
\hline Crosbeak & 30 & 65 & & 4 & 6 & 10 & 2 & & & 292 & & +10 & & & & 6 & & 24 & 28 \\
\hline sbeak & 6 & 247 & 93 & 67 & 20 & 2 & 2 & & & 10 & 7 & 30 & +22 & & & +7 & & 49 & 52 \\
\hline Rosy Finch & & & & & & & & & & & & & & +1 & & & & 1 & 4 \\
\hline dpoll & & 16 & 1 & & & 2 & & & & 1 & 27 & & & & & & & 18 & 19 \\
\hline Redpoll & 30 & 2453 & 229 & 285 & 93 & 41 & 17 & 9 & 15 & 26 & 463 & 4 & +17 & 2 & & 3 & & 51 & 53 \\
\hline d Junco & & +1 & & & & & & & & 1 & & & & & & & & 3 & 4 \\
\hline row & & 2 & & & & & & & & & & & & & & & & 2 & 2 \\
\hline Longspur & & & & & & & & & & & & & & & & & & 2 & 2 \\
\hline nting & 90 & 154 & 359 & 100 & 76 & 177 & 1 & 33 & 150 & +300 & 15 & 35 & +20 & & & 208 & 83 & 44 & 49 \\
\hline & & & & & & & & & & & & & & & & & & & \\
\hline Iy Species & 14 & 34 & 16 & 18 & 15 & 18 & 76 & 9 & 11 & 17 & 15 & 9 & 7 & 8 & 6 & 12 & 3 & & \\
\hline riod Species & 14 & 37 & 16 & 19 & 15 & 19 & 21 & 15 & 11 & 20 & 15 & 12 & 12 & 10 & 6 & 15 & 3 & & \\
\hline
\end{tabular}

h, 1978. 36(1) 
1. Bangor

2. Battleford-North Battleford

3. Beaver Creek

4. Besnard Lake

5. Biggar

6. Big Gully Creek

7. Broadview

8. Candle Lake

9. Clarkboro

10. Cold River

11. Dalmeny

12. Dilke

13. Duck Lake

14. Duperow-Ruthilda

15. Eastend-Ravenscrag

16. Eastend-Ravenscrag

17. Endeavour

18. Endeavour

19. Feudal

20. Fort Qu'Appelle

21. Fort Walsh

22. Gardiner Dam

23. Clamis-Wiseton
24. Glaslyn-Turtle Lake

25. Grenfell

26. Hafford-Blaine Lake

27. Hawarden

28. Humboldt

29. Indian Head

30. Ituna

31. Kenaston

32. Kindersley

33. Kindersley

34. Kutawagan Lake

35. Langham

36. Last Mountain Lake

37. Leader

38. Luseland

39. MacDowall

40. Maidstone Bridge

41. Moose Jaw

42. Moose Mountain Prov. Park

43. North Battleford

44. Pike Lake

45. Pike Lake

46. Prince Albert
47. Prince Albert Nat. Park

48. Raymore

49. Regina

50. Rosetown

51. Round Lake

52. Saltcoats

53. Saskatoon

54. Scott

55. Skull Creek

56. Skull Creek

57. Somme

58. Spirit lake

59. Spring Valley

60. Squaw Rapids-Cut Beaver

61. Togo

62. Valley Centre

63. Waseca

64. Webb

65. White Bear

66. Whitebeech

67. Wolseley

68. Yorkton

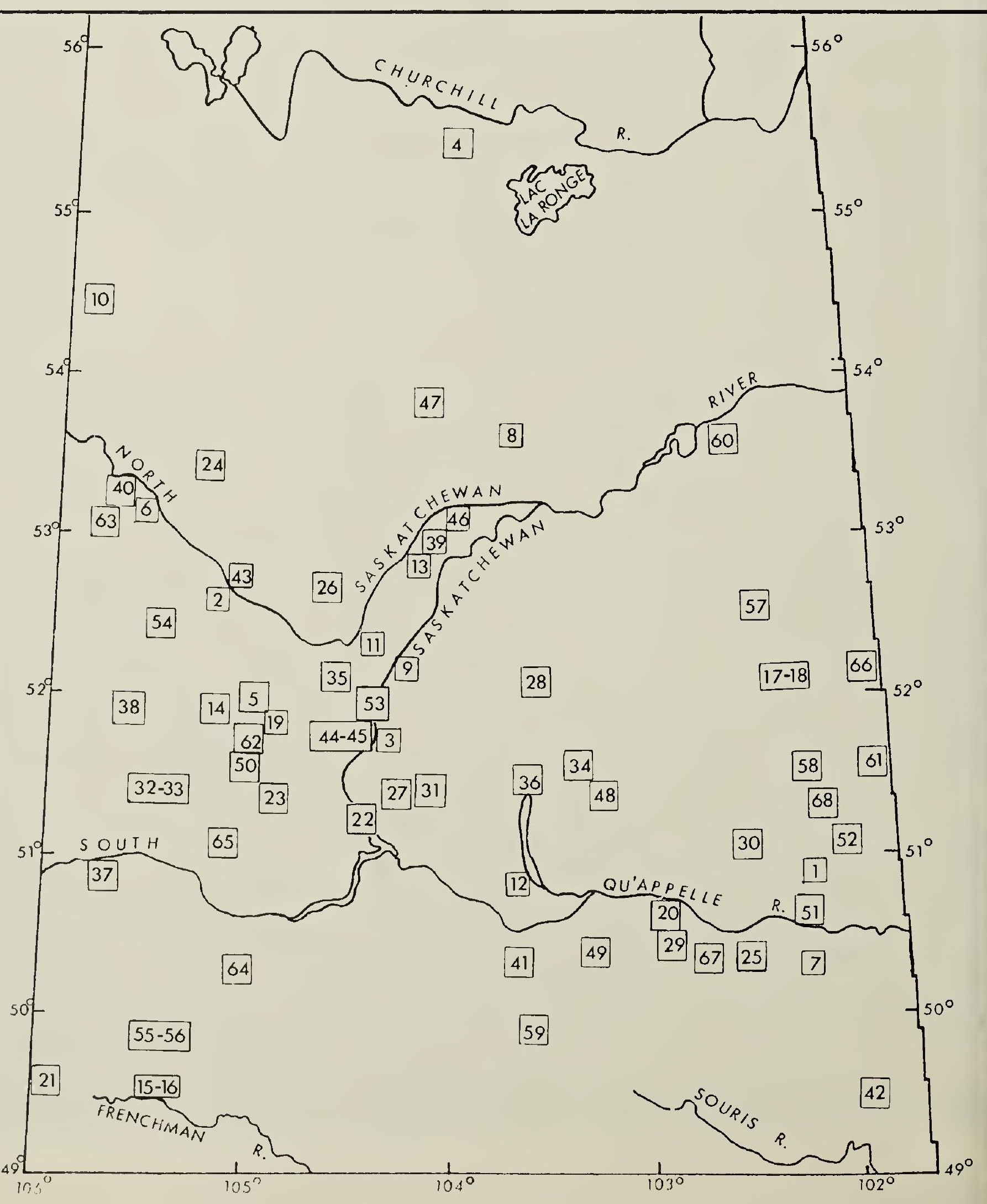


Table 4. SPECIES RECORDED FROM ONLY ONE LOCALITY

No-

Species

Locality

Map No.

$\begin{array}{ll}1 & \text { Horned Grebe } \\ 7 & \text { Lesser Scaup } \\ 1 & \text { Sharp-Shinned Hawk } \\ 1 & \text { Peregrine Falcon } \\ 1 & \text { Spruce Crouse } \\ 1 & \text { Claucous Cull } \\ 1 & \text { Barred Owl } \\ 1 & \text { Boreal Owl } \\ 1 & \text { Townsend's Solitaire } \\ 8 & \text { Golden-Crowned Kinglet } \\ 3 & \text { Redwinged Blackbird } \\ 6 & \text { Red Crossbill } \\ 1 & \text { Eagle Sp }\end{array}$

Regina 49

Regina 49

Eastend-Ravenscrag $\quad 16$

Eastend-Ravenscrag 16

Candle Lake $\quad 8$

Cold River 10

Endeavour 18

Valley Centre 62

Fort Walsh 21

Fort Walsh 21

Fort Walsh 21

Fort Walsh 21

Feudal 19

Table 5. SASKATCHEWAN TEMPERATURE AND PRECIPITATION NovemberDecember, $1977^{*}$

TEMPERATURE $\left({ }^{\circ} \mathrm{C}\right)$

Difference

Mean

Swift Current

$$
\begin{aligned}
& \text { Nov. }-5.5 \\
& \text { Dec. }-15.0
\end{aligned}
$$

from Normal

$$
-1.8
$$$$
-5.3
$$$$
-1.7
$$$$
\text { Dec. }-17.9
$$$$
-5.0
$$$$
-0.2
$$

Nov. -5.7

Dec. -18.8

$$
-5.5
$$

Nov. -6.9

$-1.0$

Battleford

Dec. -19.0

$-5.0$

$-1.2$

$-5.3$

Dec. -19.3

$$
-0.9
$$

Dec. $-21.1-4.7$

$+0.5$

$-4.1$

Dec. -20.5
PRECIPITATION (mm)

Snow on

Total Percent Ground at Snowfall Precipitation of Normal Monthend 421

48.6

$254 \%$

270

13.8

$85 \%$

80

314

26.2

$161 \%$

320

15.2

$88 \%$

$246 \%$

50

396

33.2

$62 \%$

250

10.9

$154 \%$

100

31.0

350

16.0

$62 \%$

$154 \%$

80

28.1

150

18.3

$89 \%$

$111 \%$

24.5

$76 \%$

$122 \%$

160

21.9

222

25.2

290

From Canadian Weather Review 
List of high species counts for each year. Additional species seen during count period are given in parentheses after the count-day total. Where another count recorded more species during count period than did the "high count" locality, the former is listed in square brackets.

1942/43 Nipawin 18

1943/44 Nipawin 15

1944/45 Nipawin 18 [Skull Creek (19)']

1945/46 Wallwort 20(2)

1946/47 Torch River 17

1947/48 Skull Creek 17

[Torch River $\left.(21)^{2}\right]$

1948/49 Nipawin/Fishing Lakes 25

1949/50 Nipawin 17

1950/51 Nipawin 12

[Skull Creek (19)']

1951/52 Nipawin 14 (8)

1952/53 Nipawin $24(3)$

1953/54 Yorkton 20(2)

[Somme $15(10)]$

1954/55 Yorkton 20

[Nipawin/White Gull Ck. 17 (13)]

[Saskatoon $15(13)^{3}$ ]

1955/56 Saskatoon 23

[Regina $21(7)$ ]

1956/57 Regina 21 (4)

1957/58 Regina 24 (5)

1958/59 Saskatoon 23(2)

\author{
1959/60 Regina 23 \\ 1960/61 Regina 34 (9) \\ 1961/62 Regina 37 (2) \\ 1962/63 Regina $34(8)$ \\ 1963/64 Regina 29(12) \\ 1964/65 Regina 37 (4) \\ 1965/66 Regina 31 (6) \\ 1966/67 Regina 36 \\ 1967/68 Regina 36(3) \\ 1968/69 Saskatoon 36(2) \\ 1969/70 Regina 37 (3) \\ 1970/71 Regina 33 \\ 1971/72 Regina 33 \\ 1972/73 Regina 32 (4) \\ 1973/74 Regina 40 \\ [Saskatoon 39(2)] \\ 1974/75 Saskatoon 42(3) \\ [Regina $38(14)$ ] \\ 1975/76 Regina 47 \\ 1976/77 Regina 38 (1) \\ 1977/78 Saskatoon $34(3)$ \\ Fort Walsh 34 (1)
}

'number of species observed during Christmas week

${ }^{2}$ December 20 to January 2

${ }^{3} 3$ Saskatoon counts combined

\section{ADDITIONAL COUNTS*}

\section{CANORA}

Date: December 31, 1977.

Weather: Cloudy; temperature minus $15^{\circ} \mathrm{C}$.

Area: Walked $3 \mathrm{~km}$, by car $7 \mathrm{~km}$.

Birds Seen: Sharp-tailed Grouse, 8; Gray Partridge, 10; Great Horned Owl,

*EDITOR'S NOTE: The results of the counts in Canora and Snowden were received after Mary Houston compiled the others. The Pine Siskins at Snowden raise the total species sighted this year to 67 .
1; Hairy Woodpecker, 2; Downy Woodpecker, 2; Northern 3-toed Woodpecker, 2; Blue Jay, 8: Blackcapped Chickadee, 6; White-breasted Nuthatch, 1; Evening Grosbeak, 50; Pine Grosbeak, 20. TOTAL 11 species, 110 individuals. (Add: Snowy Owl, 1; Bohemian Waxwing, 10; House Sparrow, 15; Snow Bunting, 20; all on January 2, 1978.)

Contributors: Alfred \& Vicky Tataryn.

\section{SNOWDEN}

Date: December 28, 1977.

Weather: Overcast; temperature minus $15^{\circ} \mathrm{C}$. 
Area: Walked $2 \mathrm{~km}$, by car $10 \mathrm{~km}$.

Birds Seen: Black-billed Magpie, 2; Common Raven, 3; Black-capped Chickadee, 3; House Sparrow, 6; Pine Grosbeak, 45; Common Redpoll, 60; Pine Siskin, 2; Snow Bunting, 7. TOTAL 8 species, 130 individuals.

Contributors: J. Soroka (compiler), W. Wizniuk.

\section{FORT SMITH, N.W.T}

\section{CHRISTMAS BIRD COUNT}

Date: December 27, 1977

Weather: Overcast with ice fog; temperature $-16^{\circ}$ Celsius; calm; snow depth approx. $25 \mathrm{~cm}$; daylight $0900 \mathrm{hrs}$. to 1600 hrs.

Routes Covered: Fort Smith west along
N.W.T. Highway 5 to Fox Holes Road intersection; Fort Smith south along Pine Lake Road to Salt River; Fort Smith east along Hay Camp Road to Wood Buffalo National Park Border. $160 \mathrm{~km}$ in 3.5 hours.

Birds Seen: Spruce Grouse, 1; Willow Ptarmigan, 13; Sharp-tailed Grouse, 1; Gyrfalcon, 3; Hairy Woodpecker, 1; Downy Woodpecker, 1; Northern 3toed Woodpecker, 1; Gray Jay, 25; Common Raven, 193; Pine Grosbeak, 2; Unidentified Redpolls, 60; Snow Bunting, 1; Black-capped Chickadee, 11; Boreal Chickadee, 1. TOTAL: 14 species, 314 individuals.

Contributors: Nancy \& Ian Church, Bob Lewis, Jon Lewis, Joe Benge \& Jill Schwartz, Harold Pankratz, Denis Cook, Chris Jalkotzy, Carl, Jan \& Sean Lentowicz, Bernie \& Joy Lieff, Murray Garnett, Linda \& Dan (compiler) Graham.

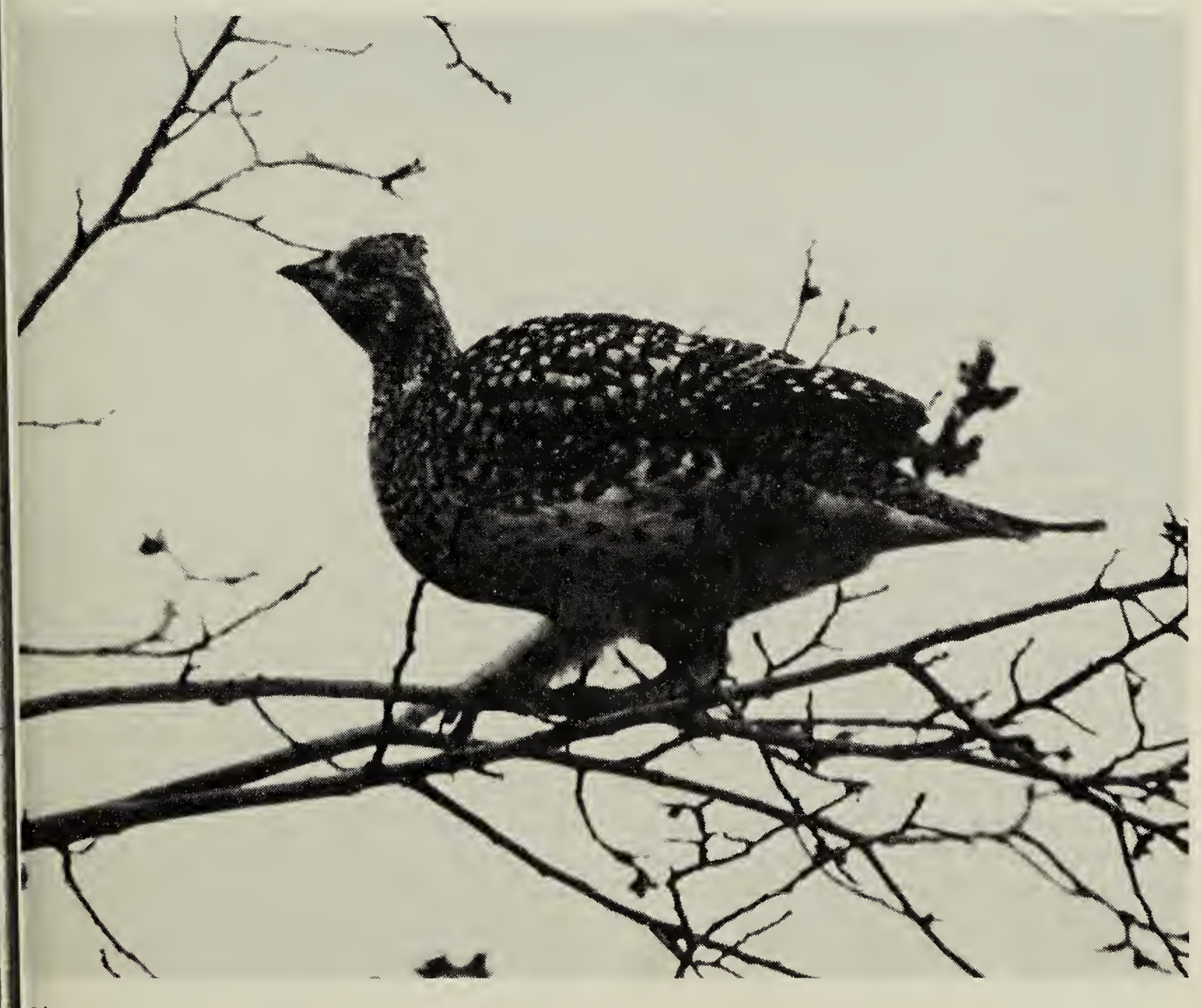

Sharp-tailed Crouse

Gary W. Seib 